\title{
Numerical solution of nonlinear elliptic systems by block monotone iterations
}

\author{
M. Al-Sultani ${ }^{1} \quad$ I. Boglaev ${ }^{2}$
}

(Received 7 February 2019; revised 4 June 2019)

\begin{abstract}
We present numerical methods for solving a coupled system of nonlinear elliptic problems, where reaction functions are quasimonotone nondecreasing. We utilize block monotone iterative methods based on the Jacobi and Gauss-Seidel methods incorporated with the upper and lower solutions method. A convergence analysis and the theorem on uniqueness of solutions are discussed. Numerical experiments are presented.
\end{abstract}

\section{Contents}

\section{Introduction}

DOI:10.21914/anziamj.v60i0.13986 gives this article, (c) Austral. Mathematical Soc. 2019. Published July 12, 2019, as part of the Proceedings of the 18th Biennial Computational Techniques and Applications Conference. ISSN 1445-8810. (Print two pages per sheet of paper.) Copies of this article must not be made otherwise available on the internet; instead link directly to the DOI for this article. 
2 Block monotone iterative methods

C81

2.1 Convergent analysis . . . . . . . . . . . . . C88

2.2 Uniqueness of a solution . . . . . . . . . . . . . . C90

3 Numerical experiments

C91

References

C93

\section{Introduction}

Several problems in the chemical, physical and engineering sciences are characterized by coupled systems of nonlinear elliptic equations [3]. In this article, we construct block monotone iterative methods for solving the coupled system of nonlinear elliptic equations

$$
\begin{aligned}
& -L_{\alpha} u_{\alpha}(x, y)+f_{\alpha}(x, y, u)=0, \quad(x, y) \in \omega, \quad \alpha=1,2, \\
& w=\{(x, y): 0<x<1,0<y<1\}, \\
& u(x, y)=g(x, y), \quad(x, y) \in \partial \omega,
\end{aligned}
$$

where $u=\left(u_{1}, u_{2}\right), f=\left(f_{1}, f_{2}\right), g=\left(g_{1}, g_{2}\right)$, and $\partial \omega$ is the boundary of $\omega$. The differential operators $\mathrm{L}_{\alpha}, \alpha=1,2$, are defined by

$$
\mathrm{L}_{\alpha} \mathrm{u}_{\alpha}(x, y) \equiv \varepsilon_{\alpha}\left(\mathrm{u}_{\alpha, x x}+\mathrm{u}_{\alpha, y y}\right),
$$

where $\varepsilon_{\alpha}$ with $\alpha=1,2$, are positive constants. It is assumed that the functions $f_{\alpha}$ and $g_{\alpha}, \alpha=1,2$, are smooth in their respective domains.

Block monotone iterative methods, based on the method of upper and lower solutions, have only been used for solving nonlinear scalar elliptic equations [1, $2,4]$. The basic idea of the block monotone iterative methods is to decompose a two dimensional problem into a series of one dimensional two-point boundary value problems. Each of the one dimensional problems can be solved efficiently by a standard computational scheme such as the Thomas algorithm. 
In this article we construct and investigate block monotone iterative methods based on the Jacobi and Gauss-Seidel methods for solving coupled systems of nonlinear elliptic equations with quasimonotone nondecreasing reaction functions $f_{\alpha}$ with $\alpha=1,2$.

In Section 2 we consider a nonlinear difference scheme which approximates the nonlinear elliptic problem (1) and describe the construction of the block monotone Jacobi and Gauss-Seidel iterative methods. A convergence analysis of the block monotone Jacobi and Gauss-Seidel iterative methods is discussed. The theorem on uniqueness of a solution to the nonlinear difference scheme is proved. Section 3 presents numerical experiments.

\section{Block monotone iterative methods}

On $\bar{\omega}=\omega \cup \partial \omega$ we introduce a rectangular mesh $\bar{\omega}^{h}=\bar{\omega}^{\text {hx }} \times \bar{\omega}^{\text {hy }}=\omega^{h} \cup \partial \omega^{h}$ where $\partial \omega^{h}$ is the boundary of the mesh $\omega^{h}$ and

$$
\begin{aligned}
& \bar{\omega}^{\text {hx }}=\left\{x_{i}, i=0,1, \ldots, N_{x} ; \quad x_{0}=0, \quad x_{N_{x}}=1 ; \quad h_{x}=x_{i+1}-x_{i}\right\}, \\
& \bar{\omega}^{\text {hy }}=\left\{y_{j}, j=0,1, \ldots, N_{y} ; \quad y_{0}=0, \quad y_{N_{y}}=1 ; \quad h_{y}=y_{j+1}-y_{j}\right\} .
\end{aligned}
$$

For a mesh function $\mathrm{U}\left(\mathrm{p}_{\mathrm{ij}}\right)=\left(\mathrm{U}_{1}\left(\mathrm{p}_{\mathrm{ij}}\right), \mathrm{U}_{2}\left(\mathrm{p}_{\mathrm{ij}}\right)\right)$ with $\mathrm{p}_{\mathrm{ij}}=\left(x_{i}, y_{j}\right) \in \bar{\omega}^{\mathrm{h}}$ we use the difference scheme

$$
\begin{aligned}
& \mathcal{L}_{\alpha, i j} u_{\alpha}\left(p_{i j}\right)+f_{\alpha}\left(p_{i j}, u\right)=0, \quad p_{i j} \in \omega^{h}, \quad \alpha=1,2, \\
& u\left(p_{i j}\right)=g\left(p_{i j}\right), \quad p_{i j} \in \partial \omega^{h},
\end{aligned}
$$

The linear difference operators $\mathcal{L}_{\alpha}$ are defined by

$$
\mathcal{L}_{\alpha, i j} u_{\alpha}\left(p_{i j}\right)=-\varepsilon_{\alpha}\left(D_{x}^{2} u_{\alpha}\left(p_{i j}\right)+D_{y}^{2} u_{\alpha}\left(p_{i j}\right)\right),
$$


where $D_{x}^{2} U_{\alpha}\left(p_{i j}\right)$ and $D_{y}^{2} U_{\alpha}\left(p_{i j}\right)$ for $\alpha=1,2$ are the central difference approximations to the second derivatives:

$$
\begin{aligned}
& D_{\chi}^{2} u_{\alpha}\left(p_{i j}\right)=\frac{u_{\alpha, i-1, j}-2 u_{\alpha, i j}+u_{\alpha, i+1, j}}{h_{\chi}^{2}}, \\
& D_{y}^{2} u_{\alpha}\left(p_{i j}\right)=\frac{u_{\alpha, i, j-1}-2 u_{\alpha, i j}+u_{\alpha, i, j+1}}{h_{y}^{2}}, \quad u_{\alpha, i j} \equiv u_{\alpha}\left(p_{i j}\right) .
\end{aligned}
$$

The vector mesh functions $\widetilde{\mathrm{U}}$ and $\widehat{\mathrm{U}}$ are ordered upper and lower solutions, respectively, of (2) which satisfy the inequalities

$$
\begin{aligned}
& \widetilde{\mathrm{U}}_{\alpha}\left(p_{i j}\right) \geqslant \widehat{\mathrm{u}}_{\alpha}\left(p_{i j}\right), \quad p_{i j} \in \bar{\omega}^{h}, \\
& \mathcal{L}_{\alpha, i j} \widehat{\mathrm{u}}_{\alpha}\left(p_{i j}\right)+f_{\alpha}\left(p_{i j}, \widehat{\mathrm{u}}\right) \leqslant 0 \leqslant \mathcal{L}_{\alpha, i j} \widetilde{\mathrm{u}}_{\alpha}\left(p_{i j}\right)+f_{\alpha}\left(p_{i j}, \widetilde{\mathrm{u}}\right), \quad p_{i j} \in \omega^{h}, \\
& \widehat{\mathrm{u}}_{\alpha}\left(p_{i j}\right) \leqslant g_{\alpha}\left(p_{i j}\right) \leqslant \widetilde{\mathrm{u}}_{\alpha}\left(p_{i j}\right), \quad p_{i j} \in \partial \omega^{h}, \quad \alpha=1,2 .
\end{aligned}
$$

For a given pair of ordered upper and lower solutions $\widetilde{\mathrm{U}}$ and $\widehat{\mathrm{U}}$ we define the sector

$$
\langle\widehat{u}, \widetilde{u}\rangle=\left\{u\left(p_{i j}\right): \widehat{u}_{\alpha}\left(p_{i j}\right) \leqslant u_{\alpha}\left(p_{i j}\right) \leqslant \widetilde{u}_{\alpha}\left(p_{i j}\right), \quad p_{i j} \in \bar{\omega}^{h}, \quad \alpha=1,2\right\} .
$$

We assume that on $\langle\widehat{u}, \widetilde{\mathbf{u}}\rangle$ the vector function $f\left(p_{i j}, \mathcal{U}\right)$ in $(2)$ satisfies the constraints

$$
\begin{aligned}
&\left(f_{\alpha}\left(p_{i j}, U\right)\right)_{\mathfrak{u}_{\alpha}} \leqslant c_{\alpha}\left(p_{i j}\right), \quad u \in\langle\widehat{u}, \widetilde{U}\rangle, \quad \alpha=1,2, \\
&-\left(f_{\alpha}\left(p_{i j}, u\right)\right)_{u_{\alpha^{\prime}}} \geqslant 0, \quad u \in\langle\widehat{u}, \widetilde{u}\rangle, \quad \alpha^{\prime} \neq \alpha, \quad \alpha, \alpha^{\prime}=1,2,
\end{aligned}
$$

for $p_{i j} \in \bar{\omega}^{h}$ and where $\left(f_{\alpha}\right)_{u_{\alpha}} \equiv \partial f_{\alpha} / \partial u_{\alpha},\left(f_{\alpha}\right)_{u_{\alpha^{\prime}}} \equiv \partial f_{\alpha} / \partial u_{\alpha^{\prime}}$ and $c_{\alpha}$ are non-negative bounded functions on $\bar{w}^{h}$. The vector function $f\left(p_{i j}, U\right)$ is quasimonotone nondecreasing on $\langle\widehat{\mathrm{U}}, \widetilde{\mathrm{U}}\rangle$ if it satisfies (5).

To construct block iterative methods we write the difference scheme (2) at an interior mesh point $p_{i j} \in \omega^{h}$ in the form

$$
\begin{aligned}
& d_{\alpha, i j} u_{\alpha, i j}-l_{\alpha, i j} u_{\alpha, i-1, j}-r_{\alpha, i j} u_{\alpha, i+1, j}-b_{\alpha, i j} u_{\alpha, i, j-1}-t_{\alpha, i j} u_{\alpha, i, j+1}= \\
& -f_{\alpha}\left(p_{i j}, u_{1, i j}, u_{2, i j}\right)+G_{\alpha, i j}^{*},
\end{aligned}
$$


where $\mathrm{G}_{\alpha}^{*}$, like the boundary function $\mathrm{g}_{\alpha}$, describes the boundary mesh points, and

$$
\begin{aligned}
& \mathrm{l}_{\alpha, i j}=\mathrm{r}_{\alpha, i j}=\frac{\varepsilon_{\alpha}}{\mathrm{h}_{x}^{2}}, \quad \mathrm{~b}_{\alpha, i j}=\mathrm{t}_{\alpha, i j}=\frac{\varepsilon_{\alpha}}{\mathrm{h}_{y}^{2}}, \\
& \mathrm{~d}_{\alpha, i j}=\mathrm{l}_{\alpha, i j}+\mathrm{r}_{\alpha, i j}+\mathrm{b}_{\alpha, i j}+\mathrm{t}_{\alpha, \mathrm{ij}}, \quad \alpha=1,2,
\end{aligned}
$$

Define column vectors and diagonal matrices by

$$
\begin{aligned}
& \mathrm{U}_{\alpha, i}=\left(\mathrm{U}_{\alpha, i, 0}, \ldots, \mathrm{U}_{\alpha, i, N_{y}}\right)^{\top}, \quad \mathrm{G}_{\alpha, i}^{*}=\left(\mathrm{G}_{\alpha, i, 1}^{*}, \ldots, \mathrm{G}_{\alpha, i, N_{y}-1}^{*}\right)^{\top}, \\
& \mathrm{F}_{\alpha, i}\left(\mathrm{U}_{1, i}, \mathrm{U}_{2, i}\right)=\left(\mathrm{f}_{\alpha, i, 1}\left(\mathrm{U}_{1, i, 1}, \mathrm{U}_{2, i, 1}\right), \ldots, \mathrm{f}_{\alpha, i, \mathrm{~N}_{y}-1}\left(\mathrm{U}_{1, i, N_{y}-1}, \mathrm{U}_{2, i, N_{y}-1}\right)\right)^{\top}, \\
& \mathrm{L}_{\alpha, i}=\operatorname{diag}\left(\mathrm{l}_{\alpha, i, 1}, \ldots, \mathrm{l}_{\alpha, i, N_{y}-1}\right), \quad \mathrm{R}_{\alpha, i}=\operatorname{diag}\left(\mathrm{r}_{\alpha, i, 1}, \ldots, \mathrm{r}_{\alpha, i, N_{y}-1}\right),
\end{aligned}
$$

for $i=0,1, \ldots, N_{x}$ and where

$$
F_{\alpha, i}\left(U_{\alpha, i}, U_{\alpha^{\prime}, i}\right)=\left\{\begin{array}{ll}
F_{1, i}\left(U_{1, i}, U_{2, i}\right), & \alpha=1, \\
F_{2, i}\left(U_{1, i}, U_{2, i}\right), & \alpha=2,
\end{array} \quad \alpha^{\prime} \neq \alpha,\right.
$$

with symmetry $F_{\alpha, i}\left(U_{\alpha, i}, U_{\alpha^{\prime}, i}\right)=F_{\alpha, i}\left(U_{\alpha^{\prime}, i}, U_{\alpha, i}\right)$. Thus, $L_{\alpha, 1} U_{\alpha, 0}$ is on the boundary and in $\mathrm{G}_{\alpha, 1}^{*}$, and $\mathrm{R}_{\alpha, \mathrm{N}_{x}-1} \mathrm{U}_{\alpha, \mathrm{N}_{x}}$ is on the boundary and in $\mathrm{G}_{\alpha, \mathrm{N}_{x}}^{*}$. Then the difference scheme (2) is written in the form

$$
\begin{aligned}
& \mathrm{A}_{\alpha, i} \mathrm{U}_{\alpha, i}-\left(\mathrm{L}_{\alpha, i} \mathrm{U}_{\alpha, i-1}+\mathrm{R}_{\alpha, i} \mathrm{U}_{\alpha, i+1}\right)=-\mathrm{F}_{\alpha, \mathrm{i}}\left(\mathrm{U}_{\alpha, \mathrm{i}}, \mathrm{U}_{\alpha^{\prime}, \mathrm{i}}\right)+\mathrm{G}_{\alpha, \mathrm{i}}^{*}, \\
& \mathrm{U}_{\mathrm{i}}=\left(\mathrm{U}_{1, i}, \mathrm{U}_{2, \mathrm{i}}\right), \quad i=1,2, \ldots, \mathrm{N}_{\mathrm{x}}-1, \quad \alpha=1,2,
\end{aligned}
$$

where $A_{\alpha, i}$ is the tridiagonal matrix with elements $d_{\alpha, i j}, l_{\alpha, i j}$ and $r_{\alpha, i j}$ with $j=0,1, \ldots, N_{y}$. The elements of the matrices $L_{\alpha, i}$ and $R_{\alpha, i}$ are the coupling coefficients of a mesh point to $U_{\alpha, i-1, j}$ and $U_{\alpha, i+1, j}$ with $j=1,2, \ldots, N_{y}-1$.

The upper $\left\{\tilde{\mathbf{u}}_{\alpha, i}^{(\mathfrak{n})}\right\}$ and lower $\left\{\hat{\mathrm{U}}_{\alpha, i}^{(\mathfrak{n})}\right\}$ sequences of solutions with number of iterations $n \geqslant 1$ are calculated by the following block Jacobi $(\eta=0)$ and Gauss-Seidel $(\eta=1)$ iterative methods:

$$
\begin{aligned}
A_{\alpha, i} Z_{\alpha, i}^{(n)}-\eta L_{\alpha, i} Z_{\alpha, i}^{(n)}+C_{\alpha, i} Z_{\alpha, i}^{(n)}= & -\mathcal{K}_{\alpha, i}\left(U_{\alpha, i}^{(n-1)}, U_{\alpha^{\prime}, i}^{(n-1)}\right), \\
\mathcal{K}_{\alpha, i}\left(U_{\alpha, i}^{(n-1)}, U_{\alpha^{\prime}, i}^{(n-1)}\right)= & A_{\alpha, i} U_{\alpha, i}^{(n-1)}-L_{\alpha, i} U_{\alpha, i-1}^{(n-1)}-R_{\alpha, i} U_{\alpha, i+1}^{(n-1)} \\
& +F_{\alpha, i}\left(U_{\alpha, i}^{(n-1)}, U_{\alpha^{\prime}, i}^{(n-1)}\right)-G_{\alpha, i}^{*},
\end{aligned}
$$


where $\alpha=1,2$ and $i=1,2, \ldots, N_{x}-1$,

$$
\begin{aligned}
& Z_{\alpha, i}^{(n)}=\left\{\begin{array}{ll}
g_{\alpha, i}-U_{\alpha, i}^{(0)}, & n=1, \\
0, & n \geqslant 2,
\end{array} \quad i=0, N_{x},\right. \\
& Z_{\alpha, i}^{(n)}=U_{\alpha, i}^{(n)}-U_{\alpha, i}^{(n-1)}, \quad \eta=0,1,
\end{aligned}
$$

where $\mathrm{U}_{\mathrm{i}}^{(\mathrm{n}-1)}=\left(\mathrm{u}_{1, \mathrm{i}}^{(\mathrm{n}-1)}, \mathrm{u}_{2, \mathrm{i}}^{(\mathrm{n}-1)}\right), \mathcal{K}_{\alpha, \mathrm{i}}\left(\mathrm{u}_{\alpha, \mathrm{i}}^{(\mathrm{n}-1)}, \mathrm{u}_{\alpha^{\prime}, \mathrm{i}}^{(\mathrm{n}-1)}\right)$ are the residuals of the difference equations (8) on $\mathrm{U}_{\alpha, i}^{(n-1)}$, and 0 is the zero column vector with $\mathrm{N}_{x}-1$ components. The matrices $\mathrm{C}_{\alpha, i}$ are the diagonal matrices $\operatorname{diag}\left(\mathbf{c}_{\alpha, i, 1}, \ldots, c_{\alpha, i, N_{y}-1}\right)$ where the $c_{\alpha}=c_{\alpha}\left(p_{i j}\right)$ are defined in (4).

The mean-value theorem for vector-valued functions is

$$
\begin{gathered}
F_{\alpha, i}\left(U_{\alpha, i}, U_{\alpha^{\prime}, i}\right)-F_{\alpha, i}\left(V_{\alpha, i}, U_{\alpha^{\prime}, i}\right)=\left(F_{\alpha, i}\left(Y_{\alpha, i}, U_{\alpha^{\prime}, i}\right)\right)_{u_{\alpha}}\left[u_{\alpha, i}-V_{\alpha, i}\right], \\
F_{\alpha, i}\left(U_{\alpha, i}, U_{\alpha^{\prime}, i}\right)-F_{\alpha, i}\left(U_{\alpha, i}, V_{\alpha^{\prime}, i}\right)=\left(F_{\alpha, i}\left(U_{\alpha, i}, Y_{\alpha^{\prime}, i}\right)\right)_{u_{\alpha^{\prime}}}\left[u_{\alpha^{\prime}, i}-V_{\alpha^{\prime}, i}\right],
\end{gathered}
$$

where the $Y_{\alpha, i}$ lie between $U_{\alpha, i}$ and $V_{\alpha, i}$, and the $Y_{\alpha^{\prime}, i}$ lie between $U_{\alpha^{\prime}, i}$ and $V_{\alpha^{\prime}, i}$, for $i=1,2, \ldots, N_{x}-1, \alpha^{\prime} \neq \alpha, \alpha, \alpha^{\prime}=1,2$. The partial derivatives are the diagonal matrices

$$
\begin{aligned}
& \left(F_{\alpha, i}\right)_{\mathfrak{u}_{\alpha}}=\operatorname{diag}\left(\left(f_{\alpha, i, 1}\right)_{\mathfrak{u}_{\alpha}}, \ldots,\left(f_{\alpha, i, N_{y}-1}\right)_{\mathfrak{u}_{\alpha}}\right), \\
& \left(F_{\alpha, i}\right)_{\mathfrak{u}_{\alpha^{\prime}}}=\operatorname{diag}\left(\left(f_{\alpha, i, 1}\right)_{\mathfrak{u}_{\alpha^{\prime}}}, \ldots,\left(f_{\alpha, i, N_{y}-1}\right)_{\mathfrak{u}_{\alpha^{\prime}}}\right),
\end{aligned}
$$

where $\left(f_{\alpha, i, j}\right)_{\mathfrak{u}_{\alpha}}$ and $\left(f_{\alpha, i, j}\right)_{\mathfrak{u}_{\alpha^{\prime}}}, j=1,2, \ldots, N_{y}-1$, are calculated at $Y_{\alpha, i}$ and $Y_{\alpha^{\prime}, i}$, respectively.

Theorem 1. Assume that $\mathrm{f}_{\alpha}$ with $\alpha=1,2$ satisfies (4) and (5). Let $\tilde{\mathrm{U}}=$ $\left(\tilde{\mathrm{U}}_{1}, \tilde{\mathrm{U}}_{2}\right)$ and $\hat{\mathrm{U}}=\left(\widehat{\mathrm{U}}_{1}, \widehat{\mathrm{U}}_{2}\right)$ be ordered upper and lower solutions of (2). Then for $\mathfrak{i}=0,1, \ldots, N_{x}$ the upper sequence $\left\{\tilde{\mathbf{U}}_{\alpha, i}^{(\mathrm{n})}\right\}$ generated by (9) with $\tilde{\mathrm{U}}^{(0)}=\tilde{\mathrm{U}}$ converges monotonically from above to a maximal solution $\tilde{\mathrm{V}}$, and similarly, the lower sequence $\left\{\widehat{\mathrm{U}}_{\alpha, \mathrm{i}}^{(\mathrm{n})}\right\}$ generated by (9) with $\widehat{\mathrm{U}}^{(0)}=\widehat{\mathrm{U}}$ converges from below to a minimal solution $\widehat{\nabla}$, such that,

$$
\widehat{\mathrm{u}}_{\alpha, \mathrm{i}}^{(\mathrm{n}-1)} \leqslant \widehat{\mathrm{u}}_{\alpha, \mathrm{i}}^{(\mathrm{n})} \leqslant \widehat{\mathrm{V}}_{\alpha, \mathrm{i}} \leqslant \tilde{\mathrm{V}}_{\alpha, \mathrm{i}} \leqslant \tilde{\mathrm{u}}_{\alpha, \mathrm{i}}^{(\mathrm{n})} \leqslant \tilde{\mathrm{U}}_{\alpha, \mathrm{i}}^{(\mathrm{n}-1)},
$$

where the inequalities between vectors are in a component-wise sense, for example, $\mathrm{U}_{\alpha, \mathrm{i}} \leqslant \mathrm{V}_{\alpha, \mathrm{i}}$ implies $\mathrm{U}_{\alpha, \mathrm{ij}} \leqslant \mathrm{V}_{\alpha, \mathrm{ij}}$ for all $\mathrm{j}=0, \ldots, \mathrm{N}_{\mathrm{y}}$. 
Proof: Since $\tilde{\mathbf{U}}^{(0)}$ is an initial upper solution (3), from (9) we have $A_{\alpha, i} \tilde{Z}_{\alpha, i}^{(1)}-\mathrm{L}_{\alpha, i} \tilde{Z}_{\alpha, i-1}^{(1)}+\mathrm{C}_{\alpha, i} \tilde{Z}_{\alpha, i}^{(1)}=-\mathcal{K}_{\alpha, i}\left(\tilde{\mathrm{U}}_{\alpha, i}^{(0)}, \tilde{\mathrm{U}}_{\alpha^{\prime}, \mathrm{i}}^{(0)}\right), \quad i=1,2, \ldots, \mathrm{N}_{\alpha}-1$,

$\tilde{Z}_{\alpha, i}^{(1)} \leqslant 0, \quad i=0, N_{x}, \quad \alpha=1,2$.

Since $L_{\alpha, i} \geqslant O$ and $\left(A_{\alpha, i}+C_{\alpha, i}\right)^{-1} \geqslant O$ (Corollary 3.20, [6]) where $O$ is the $\left(N_{y}-1\right) \times\left(N_{y}-1\right)$ null matrix, for $i=1$ in (12) and $\tilde{Z}_{\alpha, 0}^{(1)} \leqslant 0$, we conclude that $\tilde{Z}_{\alpha, 1}^{(1)} \leqslant 0$. For $i=2$ in (12), using $L_{\alpha, 2} \geqslant 0$ and $\tilde{Z}_{\alpha, 1}^{(1)} \leqslant 0$, we obtain $\tilde{Z}_{\alpha, 2}^{(1)} \leqslant 0$. Thus, by induction on $i$ we prove that

$$
\tilde{Z}_{\alpha, i}^{(1)} \leqslant 0, \quad i=0,1, \ldots, N_{x}, \quad \alpha=1,2 .
$$

Similarly, we can prove that

$$
\Sigma_{\alpha, i}^{(1)} \geqslant 0, \quad i=0,1, \ldots, N_{x}, \quad \alpha=1,2 .
$$

We now prove that

$$
\widehat{\mathrm{U}}_{\alpha, i}^{(1)} \leqslant \tilde{\mathrm{U}}_{\alpha, i}^{(1)}, \quad i=0,1, \ldots, \mathrm{N}_{x}, \quad \alpha=1,2 .
$$

Defining $W_{\alpha, i}^{(n)}=\tilde{U}_{\alpha, i}^{(\mathfrak{n})}-\widehat{U}_{\alpha, i}^{(n)}$ for $i=0,1, \ldots, N_{x}$ and $\alpha=1,2$, from (9) with $i=1,2, \ldots, N_{x}-1$ and $\alpha=1$ we have

$$
\begin{aligned}
A_{1, i} W_{1, i}^{(1)}-L_{1, i} W_{1, i-1}^{(1)}+C_{1, i} W_{1, i}^{(1)}= & C_{1, i} W_{1, i}^{(0)}+R_{1, i} W_{1, i+1}^{(0)} \\
& -\left[F_{1, i}\left(\tilde{u}_{1, i}^{(0)}, \tilde{u}_{2, i}^{(0)}\right)-F_{1, i}\left(\widehat{u}_{1, i}^{(0)}, \tilde{u}_{2, i}^{(0)}\right)\right] \\
& -\left[F_{1, i}\left(\widehat{u}_{1, i}^{(0)}, \tilde{u}_{2, i}^{(0)}\right)-F_{1, i}\left(\widehat{u}_{1, i}^{(0)}, \widehat{u}_{2, i}^{(0)}\right)\right],
\end{aligned}
$$

and for $i=0, N_{x}$ we have $W_{1, i}^{(1)}=0$. By the mean-value theorem (10), for $i=0,1, \ldots, N_{x}$ we have

$$
\begin{aligned}
& F_{1, i}\left(\tilde{u}_{1, i}^{(0)}, \tilde{u}_{2, i}^{(0)}\right)-F_{1, i}\left(\widehat{u}_{1, i}^{(0)}, \tilde{u}_{2, i}^{(0)}\right)=\left(F_{1, i}\left(Q_{1, i}^{(0)}, \tilde{u}_{2, i}^{(0)}\right)\right)_{u_{1}}\left[\tilde{u}_{1, i}^{(0)}-\widehat{u}_{1, i}^{(0)}\right], \\
& F_{1, i}\left(\widehat{u}_{1, i}^{(0)}, \tilde{u}_{2, i}^{(0)}\right)-F_{1, i}\left(\widehat{u}_{1, i}^{(0)}, \widehat{u}_{2, i}^{(0)}\right)=\left(F_{1, i}\left(\widehat{u}_{1, i}^{(0)}\right), Q_{2, i}^{(0)}\right)_{u_{2}}\left[\tilde{u}_{2, i}^{(0)}-\widehat{u}_{2, i}^{(0)}\right],
\end{aligned}
$$


where $\widehat{\mathrm{U}}_{\alpha, \mathrm{i}}^{(0)} \leqslant \mathrm{Q}_{\alpha, \mathrm{i}}^{(0)} \leqslant \tilde{\mathrm{U}}_{\alpha, \mathrm{i}}^{(0)}$ for $\alpha=1,2$, and we conclude that $\left(\mathrm{F}_{1, \mathrm{i}}\right)_{\mathfrak{u}_{1}}$ and $\left(\mathrm{F}_{1, \mathrm{i}}\right)_{\mathfrak{u}_{2}}$ satisfy (4) and (5). Now with (16) we have, for $i=1,2, \ldots, N_{x}-1$,

$$
\begin{aligned}
A_{1, i} W_{1, i}^{(1)}-L_{1, i} W_{1, i-1}^{(1)}+C_{1, i} W_{1, i}^{(1)}= & \left(C_{1, i}-\left(F_{1, i}\right)_{u_{1}}\right) W_{1, i}^{(0)} \\
& -\left(F_{1, i}\right)_{\mathfrak{u}_{2}} W_{2, i}^{(0)}+R_{1, i} W_{1, i+1}^{(0)},
\end{aligned}
$$

and $W_{1, i}^{(1)}=0$ for $i=0, N_{x}$. Now with (4) and (5), and since $W_{\alpha, i}^{(0)} \geqslant 0$ for $i=0,1, \ldots, N_{x}$ and $\alpha=1,2$, and $R_{1, i} \geqslant O$, we obtain

$$
\begin{aligned}
& A_{1, i} W_{1, i}^{(1)}+C_{1, i} W_{1, i}^{(1)} \geqslant L_{1, i} W_{1, i-1}^{(1)}, \quad i=1,2, \ldots, N_{x}-1, \\
& W_{1, i}^{(1)}=0, \quad i=0, N_{x} .
\end{aligned}
$$

Since $\left(A_{1, i}+C_{1, i}\right)^{-1} \geqslant 0$ for $i=1,2, \ldots, N_{x}-1$, with $i=1$ in (18) and $W_{1,0}^{(1)}=0$, we conclude that $W_{1,1}^{(1)} \geqslant 0$. For $i=2$ in (18), and using $L_{1,2} \geqslant 0$ and $W_{1,1}^{(1)} \geqslant 0$, we obtain $W_{1,2}^{(1)} \geqslant 0$. Thus, by induction on $i$ we prove that

$$
W_{1, i}^{(1)} \geqslant 0, \quad i=0,1, \ldots, N_{x} .
$$

By following a similar argument we can prove (15) for $\alpha=2$.

We now prove that $\tilde{\mathrm{U}}_{\alpha, i}^{(1)}$ and $\widehat{\mathrm{U}}_{\alpha, i}^{(1)}$ with $i=0,1, \ldots, \mathrm{N}_{x}$ and $\alpha=1,2$ are upper and lower solutions to (9), respectively. From (9) with $\alpha=1$ and using the mean-value theorem (10), we conclude that for $i=1,2, \ldots, N_{x}-1$,

$$
\begin{aligned}
\mathcal{K}_{1, i}\left(\tilde{\mathrm{U}}_{1, i}^{(1)}, \tilde{\mathrm{U}}_{2, i}^{(1)}\right)= & -\left(\mathrm{C}_{1, i}-\frac{\partial \mathrm{F}_{1, i}\left(\tilde{\mathrm{E}}_{1, i}^{(1)}, \tilde{\mathrm{U}}_{2, i}^{(0)}\right)}{\partial \mathrm{u}_{1}}\right) \tilde{\mathrm{Z}}_{1, i}^{(1)}+\frac{\partial \mathrm{F}_{1, i}\left(\tilde{\mathrm{U}}_{1, i}^{(0)}, \tilde{\mathrm{E}}_{2, \mathrm{i}}^{(1)}\right)}{\partial \mathrm{u}_{2}} \tilde{\mathrm{Z}}_{2, \mathrm{i}}^{(1)} \\
& -\mathrm{R}_{1, i} \tilde{\mathrm{Z}}_{1, i+1}^{(1)},
\end{aligned}
$$

where

$$
\tilde{\mathrm{U}}_{\alpha, i}^{(1)} \leqslant \tilde{\mathrm{E}}_{\alpha, \mathrm{i}}^{(1)} \leqslant \tilde{\mathrm{U}}_{\alpha, \mathrm{i}}^{(0)}, \quad i=0,1, \ldots, \mathrm{N}_{\mathrm{x}}, \quad \alpha=1,2 .
$$

From (13), (14) and (15) we conclude that $\partial \mathrm{F}_{1, i} / \partial \mathbf{u}_{1}$ and $\partial \mathrm{F}_{1, \mathrm{i}} / \partial \mathbf{u}_{2}$ satisfy (4) and (5). From (4), (5), (13) and since $\mathrm{R}_{1, \mathrm{i}} \geqslant \mathrm{O}$ we conclude that

$$
\mathcal{K}_{1, i}\left(\tilde{\mathrm{U}}_{1, i}^{(1)}, \tilde{\mathrm{U}}_{2, i}^{(1)}\right) \geqslant 0, \quad i=1,2, \ldots, \mathrm{N}_{x}-1 .
$$


Similarly,

$$
\mathcal{K}_{2, i}\left(\tilde{\mathrm{U}}_{2, i}^{(1)}, \tilde{\mathrm{U}}_{1, i}^{(1)}\right) \geqslant 0, \quad i=1,2, \ldots, \mathrm{N}_{x}-1 .
$$

From (3), (20) and (21) we conclude that $\left(\tilde{\mathbf{U}}_{1, i}^{(1)}, \tilde{\mathrm{U}}_{2, i}^{(1)}\right)$ for $\mathfrak{i}=0,1, \ldots, \mathbf{N}_{\mathrm{x}}$ is an upper solution to (2). In a similar manner we obtain

$$
\mathcal{K}_{1, i}\left(\widehat{u}_{1, i}^{(1)}, \widehat{u}_{2, i}^{(1)}\right) \leqslant 0, \quad \mathcal{K}_{2, i}\left(\widehat{u}_{2, i}^{(1)}, \widehat{u}_{1, i}^{(1)}\right) \leqslant 0, \quad i=1,2, \ldots, N_{x}-1,
$$

which means $\left(\widehat{u}_{1, i}^{(1)}, \widehat{u}_{2, i}^{(1)}\right)$ for $i=0,1, \ldots, N_{x}$ is a lower solution to (2). By induction on $\mathrm{n}$ we can prove that $\left\{\tilde{\mathrm{U}}_{\alpha, i}^{(\mathfrak{n})}\right\}$ and $\left\{\widehat{U}_{\alpha, i}^{(\mathfrak{n})}\right\}$ with $i=0,1, \ldots, \mathrm{N}_{\alpha}$ and $\alpha=1,2$ are, respectively, monotone decreasing upper and monotone increasing lower sequences of solutions.

Now we prove that the limiting functions of the upper sequence $\left\{\tilde{\mathrm{U}}_{\alpha, \mathrm{i}}^{(\mathfrak{n})}\right\}$ and lower sequence $\left\{\widehat{U}_{\alpha, i}^{(\mathfrak{n})}\right\}$ with $i=0,1, \ldots, N_{x}$ and $\alpha=1,2$ are, respectively, maximal and minimal solutions of (2). From (11) we conclude that $\lim _{n \rightarrow \infty} \tilde{\mathrm{U}}_{\alpha, i}^{(\mathfrak{n})}=\tilde{\mathrm{U}}_{\alpha, i}$ exists and

$$
\lim _{n \rightarrow \infty} \tilde{Z}_{\alpha, i}^{(n)}=0, \quad i=0,1, \ldots, N_{x}, \quad \alpha=1,2 .
$$

Similar to (19), we have

$$
\begin{aligned}
& \mathcal{K}_{1, i}\left(\tilde{\mathrm{U}}_{1, i}^{(1)}, \tilde{\mathrm{U}}_{2, \mathrm{i}}^{(1)}\right)=-\left(\mathrm{C}_{1, \mathrm{i}}-\frac{\partial \mathrm{F}_{1, \mathrm{i}}\left(\tilde{\mathrm{E}}_{1, \mathrm{i}}^{(\mathrm{n})}, \tilde{\mathrm{U}}_{2, \mathrm{i}}^{(\mathrm{n}-1)}\right)}{\partial \mathrm{u}_{1}}\right) \tilde{\mathrm{Z}}_{1, \mathrm{i}}^{(\mathrm{n})}-\mathrm{R}_{1, \mathrm{i}} \tilde{\mathrm{Z}}_{1, \mathrm{i}+1}^{(\mathrm{n})} \\
& +\frac{\partial F_{1, i}\left(\tilde{U}_{1, i}^{(n-1)}, \tilde{E}_{2, i}^{(n)}\right)}{\partial u_{2}} \tilde{Z}_{2, i}^{(n)}, \quad i=1,2, \ldots, N_{x}-1,
\end{aligned}
$$

where

$$
\tilde{\mathrm{U}}_{\alpha, i}^{(\mathrm{n})} \leqslant \tilde{\mathrm{E}}_{\alpha, \mathrm{i}}^{(\mathrm{n})} \leqslant \tilde{\mathrm{U}}_{\alpha, \mathrm{i}}^{(\mathrm{n}-1)}, \quad i=0,1, \ldots, \mathrm{N}_{\mathrm{x}}, \quad \alpha=1,2 .
$$

By taking the limit of both sides of (23), and using (13), it follows that

$$
\mathcal{K}_{1, i}\left(\tilde{\mathrm{U}}_{1, i}^{(1)}, \tilde{\mathrm{U}}_{2, \mathrm{i}}^{(1)}\right)=0, \quad i=1,2, \ldots, \mathrm{N}_{x}-1 .
$$


Similarly, we obtain

$$
\mathcal{K}_{2, i}\left(\tilde{\mathrm{U}}_{2, i}, \tilde{\mathrm{U}}_{1, \mathrm{i}}\right)=0, \quad i=1,2, \ldots, \mathrm{N}_{\mathrm{x}}-1 .
$$

From (24) and (25) we conclude that $\left(\tilde{\mathbf{U}}_{1, i}, \tilde{\mathbf{U}}_{2, i}\right)$ with $\mathfrak{i}=0,1, \ldots, \mathbf{N}_{x}$ is a maximal solution to the nonlinear difference scheme (2). In a similar manner, we can prove that

$$
\mathcal{K}_{1, i}\left(\widehat{\mathrm{U}}_{1, i}, \hat{\mathrm{u}}_{2, i}\right)=0, \quad \mathcal{K}_{2, i}\left(\widehat{\mathrm{U}}_{2, i}, \hat{\mathrm{U}}_{1, i}\right)=0, \quad i=1,2, \ldots, \mathrm{N}_{x}-1,
$$

which means that $\left(\hat{\mathrm{U}}_{1, i}, \hat{\mathrm{U}}_{2, i}\right)$ with $i=0,1, \ldots, \mathrm{N}_{\mathrm{x}}$ is a minimal solution to the nonlinear difference scheme (2).

\subsection{Convergent analysis}

Assume that the reaction functions $f_{\alpha}$ with $\alpha=1,2$ satisfy the assumptions

$$
\begin{aligned}
& 0<\hat{\mathbf{c}}_{\alpha}(x, y) \leqslant\left(f_{\alpha}(x, y, u)\right)_{\mathfrak{u}_{\alpha}} \leqslant \tilde{c}_{\alpha}(x, y), \\
& 0 \leqslant-\left(f_{\alpha}(x, y, u)\right)_{u_{\alpha^{\prime}}} \leqslant q_{\alpha \alpha^{\prime}}(x, y), \quad \alpha^{\prime} \neq \alpha, \quad \alpha, \alpha^{\prime}=1,2, \\
& \rho=\min _{\alpha=1,2}\left\{\min _{(x, y) \in \tilde{\omega}} \hat{c}_{\alpha}(x, y)\right\}>0 \text {, } \\
& 0<\beta=\max _{\alpha=1,2}\left[\max _{(x, y) \in \tilde{\omega}}\left(\frac{\mathrm{q}_{\alpha \alpha^{\prime}}(x, y)}{\hat{\mathrm{c}}_{\alpha}(x, y)}\right)\right]<1, \quad \alpha^{\prime} \neq \alpha, \quad \alpha, \alpha^{\prime}=1,2 .
\end{aligned}
$$

A stopping test for the block monotone iterative methods (9) is chosen to be

$$
\max _{\alpha=1,2}\left\|\mathcal{K}_{\alpha}\left(\mathrm{U}^{(\mathfrak{n}-1)}\right)\right\|_{\omega^{\mathrm{h}}} \leqslant \delta, \quad\left\|\mathcal{K}_{\alpha}\left(\mathrm{U}^{(\mathfrak{n}-1)}\right)\right\|_{\omega^{\mathrm{h}}}=\max _{1 \leqslant i \leqslant N_{x}-1}\left|\mathrm{~K}_{\alpha, i}\left(\mathrm{U}_{i}^{(\mathfrak{n})}\right)\right|,
$$

where $\delta$ is a prescribed accuracy.

The linear version of problem (2) is

$$
\begin{aligned}
& \mathcal{L}_{\alpha, i j} W_{\alpha}\left(p_{i j}\right)+c_{\alpha}^{*}\left(p_{i j}\right) W_{\alpha}\left(p_{i j}\right)=\Phi_{\alpha}\left(p_{i j}\right), \quad p_{i j} \in \omega^{h}, \\
& W\left(p_{i j}\right)=g\left(p_{i j}\right), \quad p_{i j} \in \partial \omega^{h}, \quad \alpha=1,2,
\end{aligned}
$$


where $W=\left(W_{1}, W_{2}\right)$ and the $c_{\alpha}^{*}$ with $\alpha=1,2$ are positive bounded functions. We give an estimate of the solution to (31) in the following lemma.

Lemma 2. The solution to (31) satisfies

$$
\left\|W_{\alpha}\right\|_{\bar{\omega}^{h}} \leqslant \max \left\{\left\|g_{\alpha}\right\|_{\partial \omega^{h}},\left\|\Phi_{\alpha} / c_{\alpha}^{*}\right\|_{\omega^{h}}\right\}, \quad \alpha=1,2,
$$

where

$$
\left\|g_{\alpha}\right\|_{\partial \omega^{h}}=\max _{p_{i j} \in \partial \omega^{h}}\left|g_{\alpha}\left(p_{i j}\right)\right|, \quad\left\|\frac{\Phi_{\alpha}}{c_{\alpha}^{*}}\right\|_{\omega^{h}}=\max _{p_{i j} \in \omega^{h}}\left|\frac{\Phi_{\alpha}\left(p_{i j}\right)}{c_{\alpha}^{*}\left(p_{i j}\right)}\right| .
$$

Samarskii [5] proves this lemma.

Theorem 3. Let assumptions (26)-(29) be satisfied. Then for the sequence $\left\{\mathrm{U}^{(\mathfrak{n})}\right\}$ generated by the block monotone iterative methods (9) we have

$$
\left\|\mathrm{U}^{\left(\mathrm{n}_{\delta}\right)}-\mathrm{U}^{*}\right\|_{\bar{\omega}^{\mathrm{h}}} \leqslant \frac{1}{(1-\beta) \rho} \delta
$$

where $\mathrm{U}^{*}$ is a solution of the nonlinear difference scheme (2) and $\mathrm{n}_{\delta}$ is the minimal number of iterations subject to (30).

Proof: The existence of a solution $\mathbf{U}^{*}$ to the nonlinear difference scheme (2) is established in Theorem 1. From (2), for $\mathbf{U}_{\alpha}^{\left(\mathfrak{n}_{\delta}\right)}$ and $\mathbf{U}_{\alpha}^{*}$, we have

$$
\begin{aligned}
& \mathcal{L}_{\alpha, i j} u_{\alpha}^{\left(n_{\delta}\right)}\left(p_{i j}\right)+f_{\alpha}\left(p_{i j}, u^{\left(n_{\delta}\right)}\right)=\mathcal{K}_{\alpha, i j}\left(u_{\alpha, i j}^{\left(n_{\delta}-1\right)}, u_{\alpha^{\prime}, i j}^{\left(n_{\delta}-1\right)}\right), \quad p_{i j} \in \omega^{h}, \\
& u_{\alpha, i j}^{\left(n_{\delta}\right)}\left(p_{i j}\right)=g_{\alpha}\left(p_{i j}\right), \quad p_{i j} \in \partial \omega^{h}, \quad \alpha=1,2, \\
& \mathcal{L}_{\alpha, i j} u_{\alpha}^{*}\left(p_{i j}\right)+f_{\alpha}\left(p_{i j}, u^{*}\right)=0, \quad p_{i j} \in \omega^{h}, \\
& u_{\alpha}^{*}\left(p_{i j}\right)=g_{\alpha}\left(p_{i j}\right), \quad p_{i j} \in \partial \omega^{h}, \quad \alpha=1,2 .
\end{aligned}
$$

Letting $W_{\alpha}^{(\mathfrak{n})}=\mathrm{U}_{\alpha}^{(\mathfrak{n})}-\mathrm{U}_{\alpha}^{*}$ for $\alpha=1,2$ and using the mean-value theorem, we obtain

$$
\begin{aligned}
& \mathcal{L}_{\alpha, i j} W_{\alpha}^{\left(n_{\delta}\right)}\left(p_{i j}\right)+\left(f_{\alpha}\left(p_{i j}, H_{\alpha}^{\left(n_{\delta}\right)}\right)\right)+u_{\alpha} W_{\alpha}^{\left(n_{\delta}\right)}\left(p_{i j}\right)= \\
& -\left(f_{\alpha}\left(p_{i j}, H_{\alpha^{\prime}}^{\left(n_{\delta}\right)}\right)\right)_{u_{\alpha^{\prime}}} W_{\alpha^{\prime}}^{\left(\mathfrak{n}_{\delta}\right)}\left(p_{i j}\right)+\mathcal{K}_{\alpha, i j}\left(u_{\alpha, i j}^{\left(n_{\delta}-1\right)}, u_{\alpha^{\prime}, i j}^{\left(n_{\delta}-1\right)}\right), \quad p_{i j} \in \omega^{h}, \\
& W_{\alpha, i j}^{\left(n_{\delta}\right)}\left(p_{i j}\right)=0, \quad p_{i j} \in \partial \omega^{h}, \quad \alpha^{\prime} \neq \alpha, \quad \alpha, \alpha^{\prime}=1,2,
\end{aligned}
$$


where $\mathrm{H}_{\alpha}^{\left(\mathfrak{n}_{\S}\right)}$ lies between $\mathrm{U}_{\alpha}^{\left(\mathfrak{n}_{\S}\right)}$ and $\mathrm{U}_{\alpha}^{*}$ for $\alpha=1,2$. Using the maximum principle (32) we conclude that

$$
\begin{aligned}
\left\|W_{\alpha}^{\left(\mathfrak{n}_{\delta}\right)}\right\|_{\bar{\omega}^{h}} \leqslant & \left\|\mathcal{K}_{\alpha}\left(\mathrm{U}^{\left(\mathfrak{n}_{\delta}\right)}\right)\left[\left(\mathrm{f}_{\alpha}\left(\mathrm{H}_{\alpha}^{(\mathfrak{n})}\right)\right)_{\mathfrak{u}_{\alpha}}\right]^{-1}\right\|_{\omega^{h}} \\
& +\left\|\left(\mathrm{f}_{\alpha}\left(\mathrm{H}_{\alpha^{\prime}}^{\left(\mathfrak{n}_{\delta}\right)}\right)\right)_{\mathfrak{u}_{\alpha^{\prime}}} /\left(\mathrm{f}_{\alpha}\left(\mathrm{H}_{\alpha}^{\left(\mathfrak{n}_{\delta}\right)}\right)\right)_{\mathfrak{u}_{\alpha}}\right\|_{\omega^{h}}\left\|W_{\alpha^{\prime}}^{\left(\mathfrak{n}_{\delta}\right)}\right\|_{\omega^{h}} .
\end{aligned}
$$

Letting $\mathcal{W}^{\left(\mathfrak{n}_{\delta}\right)}=\max _{\alpha=1,2}\left\|\mathcal{W}_{\alpha}^{\left(\mathfrak{n}_{\delta}\right)}\right\|_{\overline{\boldsymbol{\omega}}^{\mathrm{h}}}$ and with (28) and (29) we obtain

$$
\mathcal{W}^{\left(\mathfrak{n}_{\delta}\right)} \leqslant\left(\max _{\alpha=1,2}\left\|\mathcal{K}_{\alpha}\left(\mathrm{U}^{\left(\mathrm{n}_{\delta}\right)}\right)\right\|\right) \rho^{-1}+\beta \mathcal{W}^{\left(\mathrm{n}_{\delta}\right)} \text {. }
$$

Now with (30) we have (33). Thus, we prove the theorem.

\subsection{Uniqueness of a solution}

In this section we prove uniqueness of a solution of the discrete problem (2). Theorem 4. Let assumptions (26)-(29) be satisfied. Then the nonlinear difference scheme (2) has a unique solution.

Proof: To prove the uniqueness of a solution to the nonlinear difference scheme (2), because of (11), it suffices to prove that $\widehat{V}_{\alpha}=\tilde{V}_{\alpha}$, where $\widehat{V}_{\alpha}$ and $\tilde{V}_{\alpha}$ are the minimal and maximal solutions. Substituting $W_{\alpha}=\tilde{V}_{\alpha}-\widehat{V}_{\alpha}$ into (2) we have

$$
\begin{aligned}
& \mathcal{L}_{\alpha, i j} W_{\alpha}\left(p_{i j}\right)+f_{\alpha}\left(p_{i j}, \tilde{V}\right)-f_{\alpha}\left(p_{i j}, \widehat{V}\right)=0, \quad p_{i j} \in \omega^{h}, \\
& W_{\alpha}\left(p_{i j}\right)=0, \quad p_{i j} \in \partial \omega^{h}, \quad \alpha=1,2 .
\end{aligned}
$$

Using the mean-value theorem we obtain

$$
\begin{aligned}
& \left(\mathcal{L}_{\alpha, i j}+\left(f_{\alpha}\left(p_{i j}, Q_{\alpha}\right)\right)_{u_{\alpha}}\right) W_{\alpha}\left(p_{i j}\right)=-\left(f_{\alpha}\left(p_{i j}, Q_{\alpha^{\prime}}\right)\right)_{u_{\alpha^{\prime}}} W_{\alpha^{\prime}}\left(p_{i j}\right), \\
& p_{i j} \in \omega^{h}, \quad W_{\alpha}\left(p_{i j}\right)=0, \quad p_{i j} \in \partial \omega^{h}, \quad \alpha^{\prime} \neq \alpha, \quad \alpha, \alpha^{\prime}=1,2,
\end{aligned}
$$


where $\widehat{V}_{\alpha}\left(p_{i j}\right) \leqslant Q_{\alpha}\left(p_{i j}\right) \leqslant \tilde{V}_{\alpha}\left(p_{i j}\right.$ for $\alpha=1,2$. Using the maximum principle (32) we conclude that

$$
\begin{aligned}
\left\|W_{\alpha}\right\|_{\tilde{\omega}^{h}} & \leqslant\left\|\left(f_{\alpha}\left(Q_{\alpha^{\prime}}\right)\right)_{\mathfrak{u}_{\alpha^{\prime}}} W_{\alpha^{\prime}}\left[\left(f_{\alpha}\left(Q_{\alpha}\right)\right)_{\mathfrak{u}_{\alpha}}\right]^{-1}\right\|_{\omega^{h}} \\
& \leqslant\left\|\left(f_{\alpha}\left(Q_{\alpha^{\prime}}\right)\right)_{\mathfrak{u}_{\alpha^{\prime}}}\left[\left(f_{\alpha}\left(Q_{\alpha}\right)\right)_{\mathfrak{u}_{\alpha}}\right]^{-1}\right\|_{\omega^{h}}\left\|W_{\alpha^{\prime}}\right\|_{\omega^{h}} .
\end{aligned}
$$

Using (29) we obtain

$$
\left\|W_{\alpha}\right\|_{\tilde{\Omega}^{h}} \leqslant \beta\left\|W_{\alpha^{\prime}}\right\|_{\omega^{h}} .
$$

Let $W=\max _{\alpha=1,2}\left\|W_{\alpha}\right\|_{\bar{\omega}^{h}}$ so that

$$
W(1-\beta) \leqslant 0 .
$$

From (28) and since $W \geqslant 0$ we conclude that $W=0$. Thus, we prove the theorem.

As follows from Theorems 1 and 4, under assumptions (26)-(29), the sequences of solutions generated by the block Jacobi and Gauss-Seidel methods converge to the unique solution of the nonlinear difference scheme (2).

\section{$3 \quad$ Numerical experiments}

As a test problem we consider the gas-liquid interaction model [3] where reaction functions are

$$
f_{1}\left(u_{1}, u_{2}\right)=-\sigma_{1}\left(1-u_{1}\right) u_{2}, \quad f_{2}\left(u_{1}, u_{2}\right)=\sigma_{2}\left(1-u_{1}\right) u_{2},
$$

where $\boldsymbol{u}_{1} \geqslant 0$ and $\boldsymbol{u}_{2} \geqslant 0$ are concentrations of the gas and liquid, respectively, and $\sigma_{\alpha}=$ const $>0$ with $\alpha=1,2$ are reaction rates.

We choose $\varepsilon_{1}=1, \varepsilon_{2}=0.1$, the boundary conditions $g_{1}(x, y)=0$ and $\mathrm{g}_{2}(x, y)=1,(x, y) \in \partial \omega$ in $(1)$, and $\sigma_{\alpha}=1$ for $\alpha=1,2$. The pairs $\left(\tilde{\mathrm{U}}_{1}, \tilde{\mathrm{U}}_{2}\right)=(1,1)$ and $\left(\widehat{\mathrm{U}}_{1}, \widehat{\mathrm{U}}_{2}\right)=(0,0)$ are ordered upper and lower solutions. From (34) we conclude that

$$
\begin{aligned}
& \left(f_{1}\right)_{\mathfrak{u}_{1}}=u_{2} \leqslant 1, \quad-\left(f_{1}\right)_{\mathfrak{u}_{2}}=1-u_{1} \geqslant 0, \\
& \left(f_{2}\right)_{u_{2}}=1-u_{1} \leqslant 1, \quad-\left(f_{2}\right)_{\mathfrak{u}_{1}}=u_{2} \geqslant 0 .
\end{aligned}
$$


Table 1: Numerical error and order of convergence of the nonlinear scheme (2).

\begin{tabular}{l|lllll}
$\mathrm{N}$ & 8 & 16 & 32 & 64 & 128 \\
\hline $\mathrm{E}$ & 0.0071 & 0.0017 & $4.47 \times 10^{-4}$ & $1.06 \times 10^{-4}$ & $2.13 \times 10^{-5}$ \\
$\gamma$ & 1.97 & 2.01 & 2.06 & 2.32 &
\end{tabular}

Table 2: Number of iterations and CPU time for the block methods.

\begin{tabular}{|c|c|c|c|c|}
\hline $\mathrm{N}$ & 16 & 32 & 64 & 128 \\
\hline & \multicolumn{4}{|c|}{ block Jacobi method } \\
\hline \# of iterations & 397 & 1577 & 6299 & 25189 \\
\hline \multirow[t]{2}{*}{$\mathrm{CPU}(\mathrm{s})$} & 0.02 & 0.91 & 14.17 & 225.99 \\
\hline & \multicolumn{4}{|c|}{ block Gauss-Seidel method } \\
\hline$\#$ of it & 180 & 762 & 3084 & 12370 \\
\hline CPL & 0.01 & 0.47 & 7.34 & 117.62 \\
\hline
\end{tabular}

It follows that $\mathrm{f}_{\alpha}$ with $\alpha=1,2$ satisfy (4) with $c_{\alpha}=1$ and (5). Since the exact solution of the test problem is unavailable, we define the numerical error and the order of convergence of the numerical solution, respectively, as

$$
E(N)=\max _{\alpha=1,2}\left[\max _{p_{i j} \in \bar{\omega}_{h}}\left|u_{\alpha}^{\left(n_{\delta}\right)}\left(p_{i j}\right)-U_{\alpha}^{\left(n_{\delta}\right) r}\left(p_{i j}\right)\right|\right], \quad \gamma(N)=\log _{2}\left(\frac{E(N)}{E(2 N)}\right),
$$

where $\mathrm{U}_{\alpha}^{\left(\mathfrak{n}_{\delta}\right)}\left(\mathrm{p}_{\mathrm{ij}}\right)$ with $\alpha=1,2$ are the approximate solutions generated by (9), $n_{\delta}$ is the minimal number of iterations subject to (30), and $\mathrm{U}_{\alpha}^{\left(\mathfrak{n}_{\delta}\right) \mathrm{r}}\left(\mathrm{p}_{\mathrm{ij}}\right)$ with $\alpha=1,2$ are reference solutions with number of mesh points $N=512$.

Table 1 presents the error $E(N)$ and order of convergence $\gamma(N)$ for different values of $N_{x}=N_{y}=N$. This table indicates that the numerical solution of the nonlinear difference scheme (2) converges to the reference solution with second-order accuracy. The numerical and reference solutions are calculated by the block Jacobi or Gauss-Seidel methods. Tables 2 and 3 show that the block Gauss-Seidel method converges faster than the block Jacobi method, and the block monotone methods (Table 2) converge faster than the corresponding monotone Gauss-Seidel and Jacobi methods (Table 3). 
Table 3: Number of iterations and CPU time for the Jacobi and Gauss-Seidel methods.

\begin{tabular}{c|lllll}
$\mathrm{N}$ & 8 & 16 & 32 & 64 & 128 \\
\hline & \multicolumn{5}{|c}{ Jacobi method } \\
\hline \# of iterations & 190 & 771 & 3092 & 12378 & 49520 \\
CPU (s) & 0.08 & 0.11 & 1.09 & 16.15 & 261.28 \\
\hline & \multicolumn{5}{|c}{ Gauss-Seidel method } \\
\hline \# of iterations & 97 & 388 & 1548 & 6191 & 24762 \\
CPU (s) & 0.12 & 0.40 & 0.53 & 8.58 & 141.37
\end{tabular}

\section{References}

[1] Boglaev, I., A block monotone domain decomposition algorithm for a semilinear convection-diffusion problem, J. Comput. Appl. Math., 173(2005), 259-277. doi:10.1016/j.cam.2004.03.011 C80

[2] Boglaev, I., Monotone iterates for solving systems of semilinear elliptic equations and applications, ANZIAM J, Proceedings of the 8th Biennial Engineering Mathematics and Applications Conference, EMAC-2007, 49(2008), C591-C608. doi:10.21914/anziamj.v49i0.311 C80

[3] Pao, C. V., Nonlinear parabolic and elliptic equations, Springer-Verlag (1992). doi:10.1007/978-1-4615-3034-3 C80, C91

[4] Pao, C. V., Block monotone iterative methods for numerical solutions of nonlinear elliptic equations, Numer. Math., 72(1995), 239-262. doi:10.1007/s002110050168 C80

[5] Samarskii, A., The theory of difference schemes, CRC Press (2001). https://www.crcpress.com/The-Theory-of-Difference-Schemes/ Samarskii/p/book/9780824704681 C89

[6] Varga, R. S., Matrix iterative analysis, Springer-Verlag (2000). doi:10.1007/978-3-642-05156-2 C85 


\section{Author addresses}

1. M. Al-Sultani, School of Fundamental Sciences, Massey University, Palmerston North, New Zealand; Faculty of Education for Pure Sciences, University of Kerbala, Kerbala, Iraq.

mailto:m.al-sultani@massey.ac.nz

2. I. Boglaev, School of Fundamental Sciences, Massey University, Palmerston North, New Zealand.

mailto: i.boglaev@massey .ac.nz 